\title{
SIGN-CHANGING SOLUTIONS \\ FOR $p$-LAPLACIAN EQUATIONS \\ WITH JUMPING NONLINEARITY \\ AND THE FUČIK SPECTRUM
}

Ming Xiong - Ze-Heng Yang - Xiang-Qing LiU

AbStRaCt. We study the existence of sign-changing solutions for the $p$ Laplacian equation

$$
-\Delta_{p} u+\lambda g(x)|u|^{p-2} u=f(u), \quad x \in \mathbb{R}^{N},
$$

where $\lambda$ is a positive parameter and the nonlinear term $f$ has jumping nonlinearity at infinity and is superlinear at zero. The Fučik spectrum plays an important role in the proof. We give sufficient conditions for the existence of nontrivial Fučik spectrum.

\section{Introduction}

In this paper we are concerned with the following $p$-Laplacian equations in $\mathbb{R}^{N}$ with jumping nonlinearity:

$$
-\Delta_{p} u+\lambda g(x)|u|^{p-2} u=f(u), \quad x \in \mathbb{R}^{N},
$$

where $1<p<N, \lambda>0$ and $\Delta_{p} u=\operatorname{div}\left(|u|^{p-2} u\right)$ is the $p$-Laplacian operator.

2010 Mathematics Subject Classification. 35J92, 35J60.

Key words and phrases. Jumping; sign-changing solution; Fučik spectrum.

X.Q. Liu, corresponding author, supported by NSFC (Nos. 11101355, 11361077), Yunnan Normal University, LianDa Scholar Program and Young Academic and Technical Leaders Program (2015HB028). 
In the weak form of problem $(\mathrm{P})$ the solution $u \in W^{1, p}\left(\mathbb{R}^{N}\right)$ satisfies

$$
\int_{\mathbb{R}^{N}}|\nabla u|^{p-2} \nabla u \nabla \varphi d x+\lambda \int_{\mathbb{R}^{N}} g(x)|u|^{p-2} u \varphi d x=\int_{\mathbb{R}^{N}} f(u) \varphi d x,
$$

for all $\varphi \in W^{1, p}\left(\mathbb{R}^{N}\right)$. Problem $(\mathrm{P})$ has a variational structure given by the functional

$$
I(u)=\frac{1}{p} \int_{\mathbb{R}^{N}}\left(|\nabla u|^{p}+\lambda g(x)|u|^{p}\right) d x-\int_{\mathbb{R}^{N}} F(u) d x, \quad u \in W^{1, p}\left(\mathbb{R}^{N}\right),
$$

where $F(t)=\int_{0}^{t} f(s) d s$. Assume that the potential function $g$ satisfies the conditions:

$\left(g_{1}\right) g \in L^{\infty}\left(\mathbb{R}^{N}\right), 0 \leq g \leq 1=\lim _{|x| \rightarrow \infty} g(x)$, and $g(x) \not \equiv 1$.

$\left(\mathrm{g}_{2}\right)$ There exist $c, m, R_{0}>0$ such that $1-g(x) \geq c /|x|^{m}$, for $|x| \geq R_{0}$.

For the nonlinear term $f$ we make the following assumptions:

$\left(\mathrm{f}_{1}\right) f \in C\left(\mathbb{R}^{N}, \mathbb{R}\right), f(t) t>0$, for $t \neq 0$.

(f $\left.f_{2}\right) \lim _{|t| \rightarrow 0} \frac{f(t)}{|t|^{p-2} t}=0$.

$\left(\mathrm{f}_{3}\right)$ There exist $a, b$ such that $\lim _{t \rightarrow+\infty} \frac{f(t)}{t^{p-1}}=a, \lim _{t \rightarrow-\infty} \frac{f(t)}{|t|^{p-2} t}=b$.

$\left(\mathrm{f}_{4}\right) \frac{f(t)}{|t|^{p-2} t}$ is nondecreasing in $t>0$ and nonincreasing in $t<0$.

The condition $\left(\mathrm{f}_{3}\right)$ means that $f$ has a "jumping" nonlinearity at the infinity.

Let $L_{\lambda}: W^{1, p}\left(\mathbb{R}^{N}\right) \ni u \mapsto-\Delta_{p} u+\lambda g(x)|u|^{p-2} u \in\left(W^{1, p}\left(\mathbb{R}^{N}\right)\right)^{\prime}$, where $\left(W^{1, p}\left(\mathbb{R}^{N}\right)\right)^{\prime}$ is the dual space of $W^{1, p}\left(\mathbb{R}^{N}\right)$. By Cuesta et al. [4], the Fučik spectrum of $L_{\lambda}$ is defined as the set $\sigma\left(L_{\lambda}\right)$ of those $(a, b) \in \mathbb{R}^{2}$ for which equation (1.1) (or (1.2) in the weak form) has a nontrivial solution $u$ :

$$
-\Delta_{p} u+\lambda g(x)|u|^{p-2} u=a\left(u^{+}\right)^{p-1}-b\left(u^{-}\right)^{p-1}
$$

or $u \in W^{1, p}\left(\mathbb{R}^{N}\right)$ satisfies

$$
\begin{aligned}
\int_{\mathbb{R}^{N}}|\nabla u|^{p-2} \nabla u \nabla \varphi d x+\lambda \int_{\mathbb{R}^{N}} g(x)|u|^{p-2} u \varphi d x & \\
& =\int_{\mathbb{R}^{N}}\left(a\left(u^{+}\right)^{p-1}-b\left(u^{-}\right)^{p-1}\right) \varphi d x
\end{aligned}
$$

for all $\varphi \in W^{1, p}\left(\mathbb{R}^{N}\right)$, where $u^{ \pm}=\max ( \pm u, 0)$. Given $\theta \in(0, \pi / 2)$, we define

$$
\rho(\theta)=\inf _{u \in \Sigma} \int_{\mathbb{R}^{N}}\left(|\nabla u|^{p}+\lambda g(x)|u|^{p}\right) d x,
$$

where

$$
\Sigma=\left\{u \mid u \in W^{1, p}\left(\mathbb{R}^{N}\right), u=u^{+}-u^{-}, u^{ \pm} \neq 0, \text { and (1.4), (1.5) hold }\right\},
$$

$$
\int_{\mathbb{R}^{N}}\left(\cos \theta\left(u^{+}\right)^{p}+\sin \theta\left(u^{-}\right)^{p}\right) d x=1,
$$




$$
\frac{\int_{\mathbb{R}^{N}}\left(\left|\nabla u^{+}\right|^{p}+\lambda g(x)\left(u^{+}\right)^{p}\right) d x}{\cos \theta \int_{\mathbb{R}^{N}}\left(u^{+}\right)^{p} d x}=\frac{\int_{\mathbb{R}^{N}}\left(\left|\nabla u^{-}\right|^{p}+\lambda g(x)\left(u^{-}\right)^{p}\right) d x}{\sin \theta \int_{\mathbb{R}^{N}}\left(u^{-}\right)^{p} d x} .
$$

Theorem 1.1. Assume that $\left(\mathrm{g}_{1}\right),\left(\mathrm{g}_{2}\right),\left(\mathrm{f}_{1}\right)-\left(\mathrm{f}_{4}\right)$ hold. Let

$$
(a, b)=(\rho \cos \theta, \rho \sin \theta), \quad \theta \in(0, \pi / 2) .
$$

Assume $\rho>\rho(\theta)$, where $\rho$ is defined by (1.3). Then either

(a) $(a, b)$ belongs to the Fučik spectrum $\sigma\left(L_{\lambda}\right)$, or

(b) problem $(\mathrm{P})$ has a sign-changing solution.

To prove Theorem 1.1, we apply the method of invariant sets, see Bartsch, Liu and Weth [1], Liu and Sun [12].

We emphasize that in the assumptions of Theorem 1.1 we say no word about whether $(\rho(\theta) \cos \theta, \rho(\theta) \sin \theta)$ is a Fučik spectrum point. Define

$$
\alpha_{1}=\inf \left\{\left.\int_{\mathbb{R}^{N}}\left(|\nabla u|^{p}+\lambda g(x)|u|^{p}\right) d x\left|\int_{\mathbb{R}^{N}}\right| u\right|^{p} d x=1\right\} .
$$

It is clear that $\min (\rho(\theta) \cos \theta, \rho(\theta) \sin \theta) \geq \alpha_{1}>0$.

Suppose that the operator $L_{\lambda}$ has a positive eigenfunction $\varphi_{1}$ corresponding to the first eigenvalue $\alpha_{1}$ and satisfying the equation

$$
-\Delta_{p} u+\lambda g(x)|u|^{p-2} u=\alpha_{1}|u|^{p-2} u, \quad x \in \mathbb{R}^{N},
$$

then the lines $\left\{\alpha_{1}\right\} \times \mathbb{R}$ and $\mathbb{R} \times\left\{\alpha_{1}\right\}$ are the trivial Fučik spectrum curves. Consider the ray $l_{\theta}=\{(\rho \cos \theta, \rho \sin \theta) \mid \rho \geq 0\}$. There are two trivial Fučik spectrum points on $l_{\theta}$, that is $\left(\alpha_{1}, \alpha_{1} \tan \theta\right)$ and $\left(\alpha_{1} \cot \theta, \alpha_{1}\right)$, to which corresponding eigenfunctions are $\varphi_{1}$ and $-\varphi_{1}$, respectively. Suppose that $(\rho \cos \theta, \rho \sin \theta)$ is a nontrivial Fučik spectrum point, then the corresponding eigenfunction $u$ is signchanging and satisfies

$$
-\Delta_{p} u+\lambda g(x)|u|^{p-2} u=\rho \cos \theta\left(u^{+}\right)^{p-1}-\rho \sin \theta\left(u^{-}\right)^{p-1}, \quad x \in \mathbb{R}^{N} .
$$

By normalization, we can assume that

$$
\int_{\mathbb{R}^{N}}\left(\cos \theta\left(u^{+}\right)^{p}+\sin \theta\left(u^{-}\right)^{p}\right) d x=1 .
$$

Therefore $u \in \Sigma$ and

$$
\rho=\int_{\mathbb{R}^{N}}\left(|\nabla u|^{p}+\lambda g(x)|u|^{p}\right) d x \geq \rho(\theta) .
$$

We conclude that, if $(\rho(\theta) \cos \theta, \rho(\theta) \sin \theta)$ is a Fučik spectrum point, it should be the first nontrivial one on the ray $l_{\theta}$. We give sufficient conditions for $(\rho(\theta) \cos \theta, \rho(\theta) \sin \theta)$ to be a Fučik spectrum point. Since $\sigma\left(L_{\lambda}\right)$ is clearly symmetric with respect to the diagonal, we assume $\theta \in(0, \pi / 4)$ from now on. 
Theorem 1.2. Assume that $\left(\mathrm{g}_{1}\right)$ holds. Define for $0<\theta \leq \pi / 4$

$$
\begin{array}{r}
\Gamma(\theta)=\inf \left\{\beta \mid \frac{\int_{\mathbb{R}^{N}}\left|\nabla u^{+}\right|^{p} d x}{\int_{\mathbb{R}^{N}}(1-g(x))\left(u^{+}\right)^{p} d x}=\frac{\int_{\mathbb{R}^{N}}\left|\nabla u^{-}\right|^{p} d x}{\int_{\mathbb{R}^{N}}(\tan \theta-g(x))\left(u^{-}\right)^{p} d x}=\beta,\right. \\
\left.u \in W^{1, p}\left(\mathbb{R}^{N}\right), u=u^{+}-u^{-}, u^{ \pm} \neq 0\right\} .
\end{array}
$$

Suppose $\lambda>\Gamma(\theta)$, then $(\rho(\theta) \cos \theta, \rho(\theta) \sin \theta)$ is a Fučik spectrum point.

Since Fučik introduced the generalized notion of spectrum, now called the Fučik spectrum, many papers have been devoted to the existence of nontrivial Fučik spectrum of the $p$-Laplacian operator with $p=2$ or $p \neq 2$, on a bounded domain $\Omega$, or in the whole space $\mathbb{R}^{N}$, see for example [2], [4]. In [4] Cuesta et al. studied the Fučik spectrum of the $p$-Laplacian operator on a bounded domain $\Omega$ in $\mathbb{R}^{N}$. Given $s \in \mathbb{R}$, they considered the functional

$$
I_{s}(u)=\int_{\Omega}|\nabla u|^{p} d x-s \int_{\Omega}\left(u^{+}\right)^{p} d x
$$

restricted to

$$
S:=\left\{\left.u \in W_{0}^{1, p}(\Omega)\left|\int_{\Omega}\right| u\right|^{p} d x=1\right\} .
$$

By a min-max argument of mountain pass type, they proved the existence of a nontrivial Fučik spectrum point of the form $(s+c(s), c(s))$, where $c(s)$ is the mountain pass value. In [2] Bartsch et al. studied the Fučik spectrum for the Schrödinger operator $-\Delta+V$ in $L^{2}\left(\mathbb{R}^{N}\right)$, they followed the idea of Cuesta et al. Here we consider a minimization problem with double restrains. By using this characterization, obviously we obtain the first nontrivial Fučik spectrum point. Moreover, this definition of the Fučik spectrum is very convenient to study the existence of sign-changing solutions for the $p$-Laplacian equations with jumping nonlinearity.

\section{Existence of sign-changing solutions}

Besides problem $(\mathrm{P})$ with potential we also consider the following limit problem $\left(\mathrm{P}_{\infty}\right)$ associated to problem $(\mathrm{P})$ at infinity:

$$
-\Delta_{p} u+\lambda|u|^{p-2} u=f(u), \quad x \in \mathbb{R}^{N} .
$$

The weak form is to look for $u \in W^{1, p}\left(\mathbb{R}^{N}\right)$ satisfying

$$
\int_{\mathbb{R}^{N}}\left(|\nabla u|^{p-2} \nabla u \nabla \varphi+\lambda|u|^{p-2} u \varphi\right) d x=\int_{\mathbb{R}^{N}} f(u) \varphi d x,
$$

for all $\varphi \in W^{1, p}\left(\mathbb{R}^{N}\right)$, to which the corresponding functional is

$$
J(u)=\frac{1}{p} \int_{\mathbb{R}^{N}}\left(|u|^{p}+\lambda|u|^{p}\right) d x-\int_{\mathbb{R}^{N}} F(u) d x, \quad u \in W^{1, p}\left(\mathbb{R}^{N}\right) .
$$


The following lemmas are concerned with the existence of one-sign solutions of problems $(\mathrm{P})$ and $\left(\mathrm{P}_{\infty}\right)$, and were proved in [15], [16] for $p=2$ and [10] for general $p$.

LEMMA 2.1. Assume that $f$ satisfies $\left(\mathrm{f}_{1}\right)-\left(\mathrm{f}_{4}\right)$.

(a) If $\lambda<a$, then problem $\left(P_{\infty}\right)$ has positive solutions with the least energy

$$
d_{+}=\inf \left\{J(u) \mid u \neq 0, u \geq 0, J^{\prime}(u)=0\right\} .
$$

If $\lambda \geq a$, then problem $\left(\mathrm{P}_{\infty}\right)$ has no positive solution. For the sake of convenience we assume $d_{+}=+\infty$ in this case.

(b) If $\lambda<b$, then problem $\left(\mathrm{P}_{\infty}\right)$ has negative solutions with the least energy

$$
d_{-}=\inf \left\{J(u) \mid u \neq 0, u \leq 0, J^{\prime}(u)=0\right\} .
$$

If $\lambda \geq b$, then problem $\left(\mathrm{P}_{\infty}\right)$ has no negative solution, and we assume $d_{+}=+\infty$ in this case.

(c) If $u$ is a sign-changing solution of problem $\left(\mathrm{P}_{\infty}\right)$, then $J(u) \geq d_{+}+d_{-}$.

Lemma 2.2. Assume that $g$ and $f$ satisfy $\left(\mathrm{g}_{1}\right),\left(\mathrm{f}_{1}\right)-\left(\mathrm{f}_{4}\right)$, respectively. Assume that $a, b>0$. Then:

(a) Problem $(\mathrm{P})$ has positive and negative solutions with the least energy $c_{ \pm}=\inf \left\{I(u) \mid u \neq 0, \pm u \geq 0, I^{\prime}(u)=0\right\}$.

(b) $c_{+}<d_{+}, c_{-}<d_{-}$.

(c) If $u$ is a sign-changing solution of problem $(\mathrm{P})$, then $I(u) \geq c_{+}+c_{-}$.

To show the existence of sign-changing solutions of problem $(\mathrm{P})$, we will apply the following multiple critical points theorem.

Theorem 2.3. Let $X$ be a Banach space, $I$ be a $C^{1}$-functional defined on $X$ and satisfying the Cerami condition, that is any sequence $\left\{u_{n}\right\} \subset X$, satisfying $\left(1+\left\|u_{n}\right\|\right)\left\|I^{\prime}\left(u_{n}\right)\right\| \rightarrow 0, I(u) \rightarrow c$, possesses a convergent subsequence. Let $D_{1}$ and $D_{2}$ be open convex subsets of $X$ and

(D) $D_{1} \cap D_{2} \neq \emptyset$.

Let $A$ be an operator from $X$ to $X$ such that

(A) $A\left(\partial D_{1}\right) \subset D_{1}, A\left(\partial D_{2}\right) \subset D_{2}$.

Assume that there exist $c_{1}, c_{2}>0$ and $1<p<+\infty$ such that

$\left(\mathrm{I}_{1}\right)\left\langle I^{\prime}(u), u-A u\right\rangle \geq c_{1}\|u-A u\|^{p}$,

$\left(\mathrm{I}_{2}\right)\left\|I^{\prime}(u)\right\| \leq c_{2}\|u-A u\|^{p-1}$.

Assume that there exists a path $h:[0,1] \rightarrow X$ such that

(H) $h(0) \in D_{1}, h(1) \in D_{2}, \sup _{t \in[0,1]} I(h(t))<\inf _{u \in D_{1} \cap D_{2}} I(u)$.

Then $I$ has at least four critical points, one in each of the domains $D_{1} \cap D_{2}$, $D_{1} \backslash \bar{D}_{2}, D_{2} \backslash \bar{D}_{1}$ and $X \backslash\left(\bar{D}_{1} \cup \bar{D}_{2}\right)$, respectively. 
By $\left(\mathrm{I}_{1}\right),\left(\mathrm{I}_{2}\right)$ we can use the vector filed $u-A u$ to construct the descending flow of the functional $I$. Then condition (A) implies that $D_{1}$ and $D_{2}$ are invariant subsets of this descending flow. The method based on invariant subsets of descending flow is an efficient method in study of the existence of sign-changing solutions of nonlinear elliptic equations. We refer the reader to [1], [12] and the survey [13] for this method and many applications. Theorem 2.3 was proved in $[6]$.

We construct the sets $D_{1}, D_{2}$, the operator $A$ and the path $h$ for our functional $I$ and verify the conditions of Theorem 2.3.

Condition (D). Denote

$$
\|u\|_{\lambda}=\left(\int_{\mathbb{R}^{N}}\left(|\nabla u|^{p}+\lambda g(x)|u|^{p}\right) d x\right)^{1 / p}, \quad u \in W^{1, p}\left(\mathbb{R}^{N}\right),
$$

which is an equivalent norm of $W^{1, p}\left(\mathbb{R}^{N}\right)$. The distance in $W^{1, p}\left(\mathbb{R}^{N}\right)$ with respect to $\|\cdot\|_{\lambda}$ is denoted by $\operatorname{dist}_{\lambda}$. Let $P=\left\{u \in W^{1, p}\left(\mathbb{R}^{N}\right) \mid u \geq 0\right\}$ be the positive cone in $W^{1, p}\left(\mathbb{R}^{N}\right)$. For $\varepsilon>0$, denote

$$
D_{1}=\left\{u \mid \operatorname{dist}_{\lambda}(u, P)<\varepsilon\right\}, \quad D_{2}=\left\{u \mid \operatorname{dist}_{\lambda}(u,-P)<\varepsilon\right\} .
$$

Clearly $D_{1}, D_{2}$ are open convex subsets of $W^{1, p}\left(\mathbb{R}^{N}\right)$ and $D_{1} \cap D_{2} \neq \emptyset$.

Condition (H). We have the following lemma.

Lemma 2.4. There exists a map $\varphi: \mathbb{R}_{+}^{2} \rightarrow W^{1, p}\left(\mathbb{R}^{N}\right)$ such that:

(a) $\varphi(t, 0) \in-P, \varphi(0, s) \in P$ for $t \geq 0, s \geq 0$.

(b) $I(\varphi(t, s)) \rightarrow-\infty$, as $t+s \rightarrow+\infty$.

(c) $\sup _{t, s \geq 0} I(\varphi(t, s))<c^{*}$, where $c^{*}=\min \left(c_{+}+d_{-}, c_{-}+d_{+}\right)$.

Consequently, if we choose $R$ large enough and define $h(t)=\varphi(R \cos \pi t / 2$, $R \sin \pi t / 2$ ), then condition (H) holds.

Proof. Without lose of generality, we assume $c^{*}=c_{+}+d_{-}$. If $d_{-}=+\infty$ then $c^{*}=+\infty$. Let $(a, b)=(\rho \cos \theta, \rho \sin \theta)$. By the assumption $\rho>\rho(\theta)$, there exists a function $u \in \Sigma$ such that $u=u^{+}-u^{-}, u^{ \pm} \neq 0$ and

$$
\frac{\int_{\mathbb{R}^{N}}\left(\left|\nabla u^{+}\right|^{p}+\lambda g(x)\left(u^{+}\right)^{p}\right) d x}{\cos \theta \int_{\mathbb{R}^{N}}\left(u^{+}\right)^{p} d x}=\frac{\int_{\mathbb{R}^{N}}\left(\left|\nabla u^{-}\right|^{p}+\lambda g(x)\left(u^{-}\right)^{p}\right) d x}{\sin \theta \int_{\mathbb{R}^{N}}\left(u^{-}\right)^{p} d x}=\beta<\rho .
$$

Define $\varphi(s, t)=s u^{+}-t u^{-},(s, t) \in \mathbb{R}_{+}^{2}$. We have $I\left(s u^{+}-t u^{-}\right)=I\left(s u^{+}\right)+I\left(t u^{-}\right)$,

$$
\begin{aligned}
& \lim _{s \rightarrow+\infty} \frac{I\left(s u^{+}\right)}{s^{p}}=\frac{1}{p} \int_{\mathbb{R}^{N}}\left(\left|\nabla u^{+}\right|^{p}+\lambda g(x)\left(u^{+}\right)^{p}\right) d x-\lim _{s \rightarrow+\infty} \frac{F\left(s u^{+}\right)}{s^{p}} \\
& =\frac{1}{p} \int_{\mathbb{R}^{N}} \beta \cos \theta\left(u^{+}\right)^{p} d x-\frac{1}{p} \int_{\mathbb{R}^{N}} a\left(u^{+}\right)^{p} d x=-\frac{1}{p}(\rho-\beta) \cos \theta \int_{\mathbb{R}^{N}}\left(u^{+}\right)^{p} d x<0 .
\end{aligned}
$$


Similarly

$$
\lim _{t \rightarrow+\infty} \frac{I\left(-t u^{-}\right)}{t^{p}}=-\frac{1}{p}(\rho-\beta) \sin \theta \int_{\mathbb{R}^{N}}\left(u^{-}\right)^{p} d x<0 .
$$

Hence there exist constants $c_{1}, c_{2}$ such that

$I\left(s u^{+}-t u^{-}\right) \leq-c_{1}\left(s^{p}+t^{p}\right)+c_{2} \quad$ and $\quad I\left(s u^{+}-t u^{-}\right) \rightarrow-\infty, \quad$ as $s+t \rightarrow+\infty$.

If $d_{-}<+\infty$, then by Lemma 2.1 , problem $\left(\mathrm{P}_{\infty}\right)$ has a negative solution $v$ with $J(v)=d_{-}$. Meanwhile, by Lemma 2.2, problem $(\mathrm{P})$ has a positive solution $u$ with $I(u)=c_{+}$. Let $e$ be a vector of $\mathbb{R}^{N}$. Define $v_{R}=v(\cdot-2 R e)$ with a parameter $R$. Set $\varphi(s, t)=s u+t v_{R}$. Then there exists $c>0$ such that for $R$ large enough

$$
I\left(s u+t v_{R}\right) \leq c_{+}+d_{-}-\frac{c}{\mathbb{R}^{m}} .
$$

The above estimate was proved in [10] for asymptotically $p$-linear $p$-Laplacian equation. The same argument works here. For $R$ large enough $u$ and $v_{R}$ are almost separate. We can show (see [6], [10]) that as $s+t \rightarrow \infty$

$$
I\left(s u+t v_{R}\right)=I(s u)+J(t v)+\left(s^{p}+t^{p}\right) o(1) .
$$

Now

$$
\begin{aligned}
\lim _{s \rightarrow+\infty} \frac{I(s u)}{s^{p}} & =\frac{1}{p} \int_{\mathbb{R}^{N}}\left(|\nabla u|^{p}+\lambda g(x)|u|^{p}\right) d x-\lim _{s \rightarrow+\infty} \frac{F(s u)}{s^{p}} \\
& =\frac{1}{p} \int_{\mathbb{R}^{N}} f(u) u d x-\frac{1}{p} \int_{\mathbb{R}^{N}} a|u|^{p} d x<0,
\end{aligned}
$$

since, by $\left(\mathrm{f}_{4}\right), f(s) s \leq a s^{p}$ for $s>0$. Similarly,

$$
\begin{aligned}
\lim _{t \rightarrow+\infty} \frac{J(t v)}{t^{p}} & =\frac{1}{p} \int_{\mathbb{R}^{N}}\left(|\nabla v|^{p}+\lambda|v|^{p}\right) d x-\lim _{t \rightarrow+\infty} \frac{F(t v)}{t^{p}} \\
& =\frac{1}{p} \int_{\mathbb{R}^{N}} f(v) v d x-\frac{1}{p} \int_{\mathbb{R}^{N}} b|v|^{p} d x<0,
\end{aligned}
$$

since, again by $\left(\mathrm{f}_{4}\right), f(s) s \leq b|s|^{p}$ for $s<0$. Therefore

$$
I\left(s u+t v_{R}\right) \leq-c_{1}\left(s^{p}+t^{p}\right)+c_{2} \quad \text { for some } c_{1}, c_{2}>0
$$

and $I\left(s u+t v_{R}\right) \rightarrow-\infty$, as $s+t \rightarrow+\infty$.

Conditions $\left(\mathbf{I}_{1}\right)$ and $\left(\mathbf{I}_{2}\right)$. We define the operator $A$ (see [5]). For $p \geq 2$, $v=A u$ is defined by the following equation:

$$
\begin{aligned}
\int_{\mathbb{R}^{N}}\left(|\nabla(u-v)|^{p-2} \nabla(u-v) \nabla \varphi+\lambda g(x)|u-v|^{p-2}(u-v) \varphi\right) d x \\
=\int_{\mathbb{R}^{N}}\left(|\nabla u|^{p-2} \nabla u \nabla \varphi+\lambda g(x)|u|^{p-2} u \varphi\right) d x-\int_{\mathbb{R}^{N}} f(u) \varphi d x
\end{aligned}
$$

for all $\varphi \in W^{1, p}\left(\mathbb{R}^{N}\right)$. The right-hand side of (2.1) is nothing but $\left\langle I^{\prime}(u), \varphi\right\rangle$. Setting $\varphi=u-v$ in $(2.1)$, we obtain

$$
\left\langle I^{\prime}(u), u-v\right\rangle=\|u-v\|_{\lambda}^{p} .
$$


Also we have

$$
\begin{aligned}
& \left\|I^{\prime}(u)\right\|=\sup _{\|\varphi\|_{\lambda}=1}\left\langle I^{\prime}(u), \varphi\right\rangle \\
& =\sup _{\|\varphi\|_{\lambda}=1} \int_{\mathbb{R}^{N}}\left(|\nabla(u-v)|^{p-2} \nabla(u-v) \nabla \varphi+\lambda g(x)|u-v|^{p-2}(u-v) \varphi\right) d x \\
& \leq \sup _{\|\varphi\|_{\lambda}=1}\left\{\left(\int_{\mathbb{R}^{N}}|\nabla(u-v)|^{p} d x\right)^{(p-1) / p}\left(\int_{\mathbb{R}^{N}}|\nabla \varphi|^{p} d x\right)^{1 / p}\right. \\
& \left.\quad+\left(\int_{\mathbb{R}^{N}} \lambda g(x)|u-v|^{p} d x\right)^{(p-1) / p}\left(\int_{\mathbb{R}^{N}} \lambda g(x)|\varphi|^{p} d x\right)^{1 / p}\right\} \\
& \left.\leq \sup _{\|\varphi\|_{\lambda}=1}\left(\int_{\mathbb{R}^{N}}\left(|\nabla(u-v)|^{p}+\lambda g(x)|u-v|^{p}\right) d x\right)^{(p-1) / p}\right\} \\
& \quad \cdot\left(\int_{\mathbb{R}^{N}}\left(|\nabla \varphi|^{p}+\lambda g(x)|\varphi|^{p}\right) d x\right)^{1 / p} \\
& =\sup _{\|\varphi\|_{\lambda}=1}\|u-v\|_{\lambda}^{p-1} \cdot\|\varphi\|_{\lambda}=\|u-v\|_{\lambda}^{p-1} .
\end{aligned}
$$

For $1 \leq p<2, v=A u$ is defined by

$$
\begin{aligned}
(2.2) & \frac{1}{2} \int_{\mathbb{R}^{N}}\left(|\nabla(u-v)|^{p-2} \nabla(u-v) \nabla \varphi+\lambda g(x)|u-v|^{p-2}(u-v) \varphi\right) d x \\
+ & \frac{1}{2} \int_{\mathbb{R}^{N}}\left(\left(|\nabla u|^{p-2} \nabla u-|\nabla v|^{p-2} \nabla v\right) \nabla \varphi+\lambda g(x)\left(|u|^{p-2} u-|v|^{p-2} v\right) \varphi\right) d x \\
& =\int_{\mathbb{R}^{N}}\left(|\nabla u|^{p-2} \nabla u \nabla \varphi+\lambda g(x)|u|^{p-2} u \varphi\right) d x-\int_{\mathbb{R}^{N}} f(u) \varphi d x
\end{aligned}
$$

for all $\varphi \in W^{1, p}\left(\mathbb{R}^{N}\right)$. Take $\varphi=u-v$ in $(2.2)$. We have $\left\langle I^{\prime}(u), u-v\right\rangle \geq$ $\|u-v\|_{\lambda}^{p} / 2$. Moreover, by (2.2),

$$
\begin{aligned}
\left\|I^{\prime}(u)\right\|= & \sup _{\|\varphi\|_{\lambda}=1}\left\langle I^{\prime}(u), \varphi\right\rangle \\
\leq & \frac{1}{2}\|u-v\|_{\lambda}^{p-1}+\frac{1}{2} \sup _{\|\varphi\|_{\lambda}=1} \int_{\mathbb{R}^{N}}\left(\left(|\nabla u|^{p-2} \nabla u-|\nabla v|^{p-2} \nabla v\right) \nabla \varphi\right. \\
& \left.+\lambda g(x)\left(|u|^{p-2} u-|v|^{p-2} v\right) \varphi\right) d x \\
\leq & \frac{1}{2}\|u-v\|_{\lambda}^{p-1} \\
& +\frac{1}{2} d_{p} \sup _{\|\varphi\|_{\lambda}=1} \int_{\mathbb{R}^{N}}\left(|\nabla(u-v)|^{p-1}|\nabla \varphi|+\lambda g(x)|u-v|^{p-1}|\varphi|\right) d x \\
\leq & \frac{1}{2}\|u-v\|_{\lambda}^{p-1}+\frac{1}{2} d_{p}\|u-v\|_{\lambda}^{p-1} .
\end{aligned}
$$


Here and in what follows we need some elementary inequalities. For $p \geq 2$ and $\xi, \eta \in \mathbb{R}^{N}$ we have

$$
\begin{aligned}
\left(|\xi|^{p-2} \xi-|\eta|^{p-2} \eta, \xi-\eta\right) & \geq c_{p}|\xi-\eta|^{p} \\
\left.|| \xi\right|^{p-2} \xi-|\eta|^{p-2} \eta \mid & \leq d_{p}(|\xi|+|\eta|)^{p-2}|\xi-\eta| .
\end{aligned}
$$

For $1 \leq p \leq 2$ and $\xi, \eta \in \mathbb{R}^{N}$

$$
\begin{gathered}
\left(|\xi|^{p-2} \xi-|\eta|^{p-2} \eta, \xi-\eta\right) \geq c_{p}(|\xi|+|\eta|)^{p-2}|\xi-\eta|^{2} \\
\left.|| \xi\right|^{p-2} \xi-|\eta|^{p-2} \eta\left|\leq d_{p}\right| \xi-\left.\eta\right|^{p-1}
\end{gathered}
$$

Condition (A). Let $u \in W^{1, p}\left(\mathbb{R}^{N}\right), v=A u$. For $p \geq 2$, set $\varphi=v^{+}$in $(2.1)$.

We have

$$
\begin{aligned}
& \int_{\mathbb{R}^{N}}\left(|\nabla u|^{p-2} \nabla u-\left|\nabla\left(u-v^{+}\right)\right|^{p-2} \nabla\left(u-v^{+}\right)\right) \nabla v^{+} d x \\
& \quad+\lambda \int_{\mathbb{R}^{N}} g(x)\left(|u|^{p-2} u-\left|u-v^{+}\right|^{p-2}\left(u-v^{+}\right) v^{+}\right) d x=\int_{\mathbb{R}^{N}} f(u) v^{+} d x .
\end{aligned}
$$

By $(2.3)$,

$$
\int_{\mathbb{R}^{N}}\left(\left|\nabla v^{+}\right|^{p}+\lambda g(x)\left(v^{+}\right)^{p}\right) d x \leq c \int_{\mathbb{R}^{N}} f(u) v^{+} d x .
$$

For $1 \leq p \leq 2$, set $\varphi=v^{+}$in $(2.2)$

$$
\begin{aligned}
\frac{1}{2} \int_{\mathbb{R}^{N}}\left(|\nabla u|^{p-2} \nabla u\right. & \left.-\left|\nabla\left(u-v^{+}\right)\right|^{p-2} \nabla\left(u-v^{+}\right)\right) \nabla v^{+} d x \\
+ & \frac{\lambda}{2} \int_{\mathbb{R}^{N}} g(x)\left(|u|^{p-2} u-\left|u-v^{+}\right|^{p-2}\left(u-v^{+}\right)\right) v^{+} d x \\
& +\frac{1}{2} \int_{\mathbb{R}^{N}}\left(\left|\nabla v^{+}\right|^{p}+\lambda g(x)\left(v^{+}\right)^{p}\right) d x=\int_{\mathbb{R}^{N}} f(u) v^{+} d x
\end{aligned}
$$

The first two terms of the left-hand side of (2.6) are positive, hence we obtain (2.5) again. Now we estimate the right-hand side of (2.5). By $\left(\mathrm{f}_{1}\right)-\left(\mathrm{f}_{3}\right)$, for $p<q<p^{*}=N p /(N-p), \delta>0$

$$
\begin{aligned}
& \int_{\mathbb{R}^{N}} f(u) v^{+} d x \leq \int_{\mathbb{R}^{N}} f\left(u^{+}\right) v^{+} d x \\
& \quad \leq \delta \int_{\mathbb{R}^{N}}\left(u^{+}\right)^{p-1} v^{+} d x+c_{\delta} \int_{\mathbb{R}^{N}}\left(u^{+}\right)^{q-1} v^{+} d x \\
& \quad \leq \delta\left|u^{+}\right|_{p}^{p-1}\left|v^{+}\right|_{p}+c_{\delta}\left|u^{+}\right|_{q}^{q-1}\left|v^{+}\right|_{q} \\
& \quad=\delta \operatorname{dist}_{L^{p}}^{p-1}(u,-P) \operatorname{dist}_{L^{p}}(v,-P)+c_{\delta} \operatorname{dist}_{L^{q}}^{q-1}(u,-P) \operatorname{dist}_{L^{q}}(v,-P) \\
& \quad \leq c \delta \operatorname{dist}_{\lambda}^{p-1}(u,-P) \operatorname{dist}_{\lambda}(v,-P)+c_{\delta} \operatorname{dist}_{\lambda}^{q-1}(u,-P) \operatorname{dist}_{\lambda}(v,-P) .
\end{aligned}
$$

By (2.5) and (2.7),

$$
\operatorname{dist}_{\lambda}^{p-1}(v,-P) \leq c \delta \operatorname{dist}_{\lambda}^{p-1}(u,-P)+c_{\delta} \operatorname{dist}_{\lambda}^{q-1}(u,-P) .
$$


Take $\delta, \varepsilon_{0}>0$ such that $c \delta<(1 / 2)^{p}$ and $c_{\delta} \varepsilon_{0}^{q-p}<(1 / 2)^{p}$. Then, for $\varepsilon<\varepsilon_{0}$ and $u \in \bar{D}_{2}=\left\{u \mid \operatorname{dist}_{\lambda}(u,-P)<\varepsilon\right\}$, we have

$$
\operatorname{dist}_{\lambda}(v,-P) \leq \frac{1}{2} \operatorname{dist}_{\lambda}(u,-P),
$$

hence $A u \in D_{2}$. In particular $A\left(\partial D_{2}\right) \subset D_{2}$. Similarly $A\left(\partial D_{1}\right) \subset D_{1}$.

Continuity of the operator $A$. Let $u_{1}, u_{2} \in W^{1, p}\left(\mathbb{R}^{N}\right)$ and $v_{1}=A u_{1}$, $v_{2}=A u_{2}$. For $p \geq 2$, by (2.1), we have

$$
\begin{array}{r}
\int_{\mathbb{R}^{N}}\left(\left|\nabla\left(u_{1}-v_{1}\right)\right|^{p-2} \nabla\left(u_{1}-v_{1}\right)-\left|\nabla\left(u_{2}-v_{2}\right)\right|^{p-2} \nabla\left(u_{2}-v_{2}\right)\right) \nabla \varphi d x \\
+\lambda \int_{\mathbb{R}^{N}} g(x)\left(\left|u_{1}-v_{1}\right|^{p-2}\left(u_{1}-v_{1}\right)-\left|u_{2}-v_{2}\right|^{p-2}\left(u_{2}-v_{2}\right)\right) \varphi d x \\
=\left\langle I^{\prime}\left(u_{1}\right)-I^{\prime}\left(u_{2}\right), \varphi\right\rangle,
\end{array}
$$

for all $\varphi \in W^{1, p}\left(\mathbb{R}^{N}\right)$. Set $\varphi=\left(u_{1}-v_{1}\right)-\left(u_{2}-v_{2}\right)$ in (2.8). By (2.3), we have

$$
\begin{aligned}
\left\|\left(u_{1}-v_{1}\right)-\left(u_{2}-v_{2}\right)\right\|_{\lambda}^{p} & \leq c\left\langle I^{\prime}\left(u_{1}\right)-I^{\prime}\left(u_{2}\right),\left(u_{1}-v_{1}\right)-\left(u_{2}-v_{2}\right)\right\rangle \\
& \leq c\left\|I^{\prime}\left(u_{1}\right)-I^{\prime}\left(u_{2}\right)\right\|\left\|\left(u_{1}-v_{1}\right)-\left(u_{2}-v_{2}\right)\right\|_{\lambda},
\end{aligned}
$$

hence

$$
\left\|v_{1}-v_{2}\right\|_{\lambda} \leq\left\|u_{1}-u_{2}\right\|_{\lambda}+c\left\|I^{\prime}\left(u_{1}\right)-I^{\prime}\left(u_{2}\right)\right\|^{1 /(p-1)} .
$$

For $1 \leq p<2$, by $(2.2)$,

$$
\begin{aligned}
\frac{1}{2} \int_{\mathbb{R}^{N}}\left(\left|\nabla\left(u_{1}-v_{1}\right)\right|^{p-2} \nabla\left(u_{1}-v_{1}\right)\right. \\
\left.\quad-\left|\nabla\left(u_{2}-v_{2}\right)\right|^{p-2} \nabla\left(u_{2}-v_{2}\right)\right) \nabla\left(u_{1}-v_{1}-u_{2}+v_{2}\right) d x \\
\quad+\frac{\lambda}{2} \int_{\mathbb{R}^{N}} g(x)\left(\left|u_{1}-v_{1}\right|^{p-2}\left(u_{1}-v_{1}\right)\right. \\
\left.\quad-\left|u_{2}-v_{2}\right|^{p-2}\left(u_{2}-v_{2}\right)\right)\left(u_{1}-v_{1}-u_{2}+v_{2}\right) d x \\
\quad+\frac{1}{2} \int_{\mathbb{R}^{N}}\left(\left|\nabla v_{2}\right|^{p-2} \nabla v_{2}-\left|\nabla v_{1}\right|^{p-2} \nabla v_{1}\right) \nabla\left(v_{2}-v_{1}\right) d x \\
\quad+\frac{\lambda}{2} \int_{\mathbb{R}^{N}} g(x)\left(\left|v_{2}\right|^{p-2} v_{2}-\left|v_{1}\right|^{p-2} v_{1}\right)\left(v_{2}-v_{1}\right) d x \\
=\frac{1}{2} \int_{\mathbb{R}^{N}}\left(\left|\nabla\left(u_{1}-v_{1}\right)\right|^{p-2} \nabla\left(u_{1}-v_{1}\right)\right. \\
\left.\quad-\left|\nabla\left(u_{2}-v_{2}\right)\right|^{p-2} \nabla\left(u_{2}-v_{2}\right)\right) \nabla\left(u_{1}-u_{2}\right) d x \\
\quad+\frac{\lambda}{2} \int_{\mathbb{R}^{N}} g(x)\left(\left|u_{1}-v_{1}\right|^{p-2}\left(u_{1}-v_{1}\right)\right. \\
\left.\quad-\left|u_{2}-v_{2}\right|^{p-2}\left(u_{2}-v_{2}\right)\right)\left(u_{1}-u_{2}\right) d x+\left\langle I_{1}^{\prime}\left(u_{1}\right)-I_{1}^{\prime}\left(u_{2}\right), v_{2}-v_{1}\right\rangle,
\end{aligned}
$$

where

$$
I_{1}(u)=\frac{1}{2 p} \int_{\mathbb{R}^{N}}\left(|\nabla u|^{p}+\lambda g(x)|u|^{p}\right) d x-\int_{\mathbb{R}^{N}} F(u) d x .
$$


We estimate the left-hand side of (2.9). By (2.4),

$$
\begin{aligned}
& \left(\int_{\mathbb{R}^{N}}\left|\nabla\left(v_{2}-v_{1}\right)\right|^{p} d x\right)^{2 / p} \\
& \leq \int_{\mathbb{R}^{N}}\left(\left|\nabla v_{2}\right|+\left|\nabla v_{1}\right|\right)^{p-2}\left|\nabla\left(v_{2}-v_{1}\right)\right|^{2} d x\left(\int_{\mathbb{R}^{N}}\left(\left|\nabla v_{2}\right|+\left|\nabla v_{1}\right|\right)^{p} d x\right)^{(2-p) / p} \\
& \leq c \int_{\mathbb{R}^{N}}\left(\left|\nabla v_{2}\right|^{p-2} \nabla v_{2}-\left|\nabla v_{1}\right|^{p-2} \nabla v_{1}\right) \nabla\left(v_{2}-v_{1}\right) d x \\
& \cdot\left(\int_{\mathbb{R}^{N}}\left(\left|\nabla v_{2}\right|+\left|\nabla v_{1}\right|\right)^{p} d x\right)^{(2-p) / p} \\
& \leq c \int_{\mathbb{R}^{N}}\left(\left|\nabla v_{2}\right|^{p-2} \nabla v_{2}-\left|\nabla v_{1}\right|^{p-2} \nabla v_{1}\right) \nabla\left(v_{2}-v_{1}\right) d x\left(\left\|u_{1}\right\|_{\lambda}+\left\|u_{2}\right\|_{\lambda}\right)^{2-p}
\end{aligned}
$$

In the above we used

$$
\|v\|_{\lambda} \leq\|u\|_{\lambda}+\|u-v\|_{\lambda} \leq\|u\|_{\lambda}+c\left\|I^{\prime}(u)\right\|^{1 /(p-1)} \leq c\|u\|_{\lambda} .
$$

In a similar way we estimate other left terms of (2.9). Next we estimate the right-hand of (2.9)

$$
\begin{aligned}
\int_{\mathbb{R}^{N}} & \left(\left|\nabla\left(u_{1}-v_{1}\right)\right|^{p-2} \nabla\left(u_{1}-v_{1}\right)-\mid \nabla\left(u_{2}-v_{2}\right) \nabla\left(u_{2}-v_{2}\right)\right) \nabla\left(u_{1}-u_{2}\right) d x \\
& \quad+\lambda \int_{\mathbb{R}^{N}} g(x)\left(\left|u_{1}-v_{1}\right|^{p-2}\left(u_{1}-v_{1}\right)-\left|u_{2}-v_{2}\right|^{p-2}\left(u_{2}-v_{2}\right)\right)\left(u_{1}-u_{2}\right) d x \\
\leq & c\left(\left\|u_{1}-v_{1}\right\|_{\lambda}^{p-1}+\left\|u_{2}-v_{2}\right\|_{\lambda}^{p-1}\right)\left\|u_{1}-u_{2}\right\|_{\lambda} \\
\leq & c\left(\left\|u_{1}\right\|_{\lambda}^{p-1}+\left\|u_{2}\right\|_{\lambda}^{p-1}\right)\left\|u_{1}-u_{2}\right\|_{\lambda} .
\end{aligned}
$$

Altogether we have

$$
\begin{aligned}
\left\|v_{2}-v_{1}\right\|_{\lambda}^{2} \leq c\left(\left\|u_{1}\right\|_{\lambda}\right. & \left.+\left\|u_{2}\right\|_{\lambda}\right)\left\|u_{1}-u_{2}\right\|_{\lambda} \\
& +c\left(\left\|u_{1}\right\|^{2-p}+\left\|u_{2}\right\|^{2-p}\right)\left\|I_{1}^{\prime}\left(u_{1}\right)-I_{1}^{\prime}\left(u_{2}\right)\right\|\left\|v_{1}-v_{2}\right\|_{\lambda}
\end{aligned}
$$

and

$$
\begin{aligned}
\left\|v_{2}-v_{1}\right\|_{\lambda} \leq c\left(\left\|u_{1}\right\|_{\lambda}^{1 / 2}+\left\|u_{2}\right\|_{\lambda}^{1 / 2}\right) & \left\|u_{1}-u_{2}\right\|_{\lambda}^{1 / 2} \\
& +c\left(\left\|u_{1}\right\|^{2-p}+\left\|u_{2}\right\|^{2-p}\right)\left\|I^{\prime}\left(u_{1}\right)-I^{\prime}\left(u_{2}\right)\right\| .
\end{aligned}
$$

At this point we have verified all assumptions of Theorem 2.3 except the Cerami condition. Since we deal with a problem in the whole space, compactness is lost. Instead of the Cerami condition, we analyze the behaviour of Cerami sequences. The proof of the following lemma is similar to the argument of Lemma 4.3 of [11] (see also [17, Theorem 5.1] or [14, Proposition 2.1]).

Lemma 2.5. Let $\left\{u_{n}\right\} \subset W^{1, p}\left(\mathbb{R}^{N}\right)$ be a Cerami sequence of the functional $I$. Then there exist $u \in W^{1, p}\left(\mathbb{R}^{N}\right), v_{i} \in W^{1, p}\left(\mathbb{R}^{N}\right),\left\{y_{n, i}\right\}_{n=1}^{\infty} \in \mathbb{R}^{N}, i=1, \ldots, k$, such that: 
(a) $u$ is a solution of problem $(\mathrm{P})$.

(b) $v_{i}, i=1, \ldots, k$, are solutions of problem $\left(\mathrm{P}_{\infty}\right)$.

(c) $\left|y_{n, i}\right| \rightarrow \infty,\left|y_{n, i}-y_{n, j}\right| \rightarrow \infty, i \neq j$, as $n \rightarrow \infty$.

(d) $u_{n}-u-\sum_{i=1}^{k} v_{i}\left(\cdot-y_{n, i}\right) \rightarrow 0$, in $W^{1, p}\left(\mathbb{R}^{N}\right)$ as $n \rightarrow \infty$.

(e) $I\left(u_{n}\right)-I(u)-\sum_{i=1}^{k} J\left(v_{i}\right) \rightarrow 0$ as $n \rightarrow \infty$.

Proof of Theorem 1.1. Since the Cerami condition does not hold, we cannot apply Theorem 2.3 directly. Anyway we can find a Cerami sequence $\left\{u_{n}\right\} \subset X \backslash\left(\bar{D}_{1} \cup \bar{D}_{2}\right)$ with $\lim _{n \rightarrow \infty} I\left(u_{n}\right)<c^{*}$. Apply Lemma 2.5 to the sequence $\left\{u_{n}\right\}$. Since $u_{n} \notin \bar{D}_{1} \cup \bar{D}_{2}$, signs of $v_{1}, v_{2}, \ldots$ are different. Then only the following several cases may occur:

(a) $u=0$.

$\left(\mathrm{a}_{1}\right)$ One of $v_{i}$, say $v_{1}$ is sign-changing.

$\left(\mathrm{a}_{2}\right)$ None of $v_{i}$ is sign-changing, then one of $v_{i}$, say $v_{1}$, is positive and another one of $v_{i}$, say $v_{2}$, is negative.

(b) $u \neq 0$.

( $\left.\mathrm{b}_{1}\right) u$ is positive, then one of $v_{i}$, say $v_{1}$, is positive or sign-changing.

$\left(\mathrm{b}_{2}\right) u$ is negative, then one of $v_{i}$, say $v_{1}$ is negative or sign-changing.

$\left(\mathrm{b}_{3}\right) u$ is sign-changing.

In all these cases but $\left(b_{3}\right)$ we will arrive at contradiction. In the cases $\left(a_{1}\right)$ and $\left(\mathrm{a}_{2}\right)$ we have

$$
c^{*}>\lim _{n \rightarrow \infty} I\left(u_{n}\right) \geq d_{+}+d_{-} .
$$

In the case $\left(b_{1}\right)$, we have

$$
c^{*}>\lim _{n \rightarrow \infty} I\left(u_{n}\right) \geq c_{+}+d_{-} .
$$

In the case $\left(b_{2}\right)$, we have

$$
c^{*}>\lim _{n \rightarrow \infty} I\left(u_{n}\right) \geq c_{-}+d_{+} .
$$

In the case $\left(\mathrm{b}_{3}\right)$, we should have $k=0$, that is $u_{n} \rightarrow u$ in $H^{1}\left(\mathbb{R}^{N}\right)$, otherwise

$$
c^{*}>\lim _{n \rightarrow \infty} I\left(u_{n}\right) \geq I(u)+\min \left(d_{+}, d_{-}\right) \geq c_{+}+c_{-}+\min \left(d_{+}, d_{-}\right) .
$$

\section{The Fučik spectrum}

In this section we study the Fučik spectrum and prove Theorem 1.2. Firstly we study the problem on bounded domains. The solutions for bounded domains will be used as the minimizing sequence of the problem on unbounded domains. 
We use the following notations.

$$
\begin{gathered}
J(u)=\int_{\mathbb{R}^{N}}\left(|\nabla u|^{p}+\lambda g(x)|u|^{p}\right) d x, \quad G(u)=\int_{\mathbb{R}^{N}}\left(\cos \theta\left(u^{+}\right)^{p}+\sin \theta\left(u^{-}\right)^{p}\right) d x, \\
R(u)=\frac{J(u)}{G(u)}=\frac{\int_{\mathbb{R}^{N}}\left(|\nabla u|^{p}+\lambda g(x)|u|^{p}\right) d x}{\int_{\mathbb{R}^{N}}\left(\cos \theta\left(u^{+}\right)^{p}+\sin \theta\left(u^{-}\right)^{p}\right) d x} .
\end{gathered}
$$

TheOREM 3.1. Let $\Omega \subset \mathbb{R}^{N}$ be a bounded domain. Define

$$
\mu_{p}:=\mu_{p}(\Omega)=\inf _{u \in \Sigma_{p}} J(u)
$$

where

$$
\begin{aligned}
\Sigma_{p}=\Sigma \cap W_{0}^{1, p}(\Omega)=\left\{u \mid u \in W_{0}^{1, p}(\Omega), u=u^{+}-u^{-}, u^{ \pm} \neq 0,\right. & \\
G(u) & \left.=1, \quad R\left(u^{+}\right)=R\left(u^{-}\right)\right\} .
\end{aligned}
$$

Then $\mu_{p}$ is achieved, and $\left(\mu_{p} \cos \theta, \mu_{p} \sin \theta\right)$ is the first nontrivial Fučik spectrum point of the operator $\left(L_{\lambda}, W_{0}^{1, p}(\Omega)\right)$ on the line $l_{\theta}$. Moreover, there exists a function $u \in \Sigma_{p}$ satisfying

$(\mathrm{E})_{p} \quad-\Delta_{p} u+\lambda g(x)|u|^{p-2} u=\mu_{p}\left(\cos \theta\left(u^{+}\right)^{p-1}-\sin \theta\left(u^{-}\right)^{p-1}\right)$

or in the weak form

$(\mathrm{E})_{p}$

$$
\begin{aligned}
\int_{\Omega}\left(|\nabla u|^{p-2} \nabla u \nabla \varphi+\lambda g(x)|u|^{p-2} u \varphi\right) d x & \\
& =\mu_{p} \int_{\Omega}\left(\cos \theta\left(u^{+}\right)^{p-1}-\sin \theta\left(u^{-}\right)^{p-1}\right) \varphi d x
\end{aligned}
$$

for all $\varphi \in W_{0}^{1, p}(\Omega)$.

We would like to point out that normalized solutions of $(\mathrm{E})_{p}$ are minimizers of problem $(\mathrm{M})_{p}$ but all minimizers are not solutions of $(\mathrm{E})_{p}$. Here is a counterexample. Let $\Omega=(0, \pi) \subset \mathbb{R}^{1}, p=2$. Consider the minimization problem

$$
\begin{aligned}
& \mu=\inf \left\{\int_{0}^{\pi}|\nabla u|^{2} d t \mid u=u^{+}-u^{-} \in H_{0}^{1}(0, \pi), u^{ \pm} \neq 0,\right. \\
& \left.\qquad \frac{\int_{0}^{\pi}\left|\nabla u^{+}\right|^{2} d t}{\int_{0}^{\pi}\left(u^{+}\right)^{2} d t}=\frac{\int_{0}^{\pi}\left|\nabla u^{-}\right|^{2} d t}{\int_{0}^{\pi}\left(u^{-}\right)^{2} d t}, \int_{0}^{\pi} u^{2} d t=1\right\} .
\end{aligned}
$$

We have a family of minimizers $\varphi_{\alpha}, \alpha \in(0, \pi / 2)$,

$$
\varphi_{\alpha}(t)= \begin{cases}\frac{2}{\sqrt{\pi}} \cos \alpha \sin 2 t & \text { for } 0 \leq t \leq \frac{\pi}{2}, \\ \frac{2}{\sqrt{\pi}} \sin \alpha \sin 2 t & \text { for } \frac{\pi}{2} \leq t \leq \pi,\end{cases}
$$


of which only one is a solution of $(\mathrm{E})_{p}$, namely the one with $\alpha=\pi / 4$. This indefiniteness motivates us to consider the following inhomogeneous problems:

$(\mathrm{M})_{q}$

$$
\mu_{q}:=\mu_{q}(\Omega)=\inf _{u \in \Sigma_{q}} J(u),
$$

where $p<q<p^{*}=N p /(N-p)$, and

$$
\begin{gathered}
\Sigma_{q}=\left\{u \mid u \in W_{0}^{1, p}(\Omega), u=u^{+}-u^{-}, u^{ \pm} \neq 0, G_{q}(u)=1, R_{q}\left(u^{+}\right)=R_{q}\left(-u^{-}\right)\right\}, \\
G_{q}(u)=\int_{\Omega}\left(\cos \theta\left(u^{+}\right)^{q}+\sin \theta\left(u^{-}\right)^{q}\right) d x, \\
R_{q}(u)=\frac{J(u)}{G_{q}(u)}=\frac{\int_{\Omega}\left(|\nabla u|^{p}+\lambda g(x)|u|^{p}\right) d x}{\int_{\Omega}\left(\cos \theta\left(u^{+}\right)^{q}+\sin \theta\left(u^{-}\right)^{q}\right) d x} .
\end{gathered}
$$

LEMMA 3.2. $\mu_{q}$ is achieved.

Proof. Let $\left\{u_{n}\right\} \in \Sigma_{q} \subset W_{0}^{1, p}(\Omega)$ be a minimizing sequence. It is bounded in $W_{0}^{1, p}(\Omega)$. Assume that $u_{n} \rightarrow u$ in $W_{0}^{1, p}(\Omega), u_{n} \rightarrow u$ in $L^{q}(\Omega)$. We have

$$
R_{q}\left(u_{n}^{+}\right)=R_{q}\left(-u_{n}^{-}\right)=R_{q}\left(u_{n}\right)=\frac{J\left(u_{n}\right)}{G_{q}\left(u_{n}\right)}=J\left(u_{n}\right) \leq c .
$$

Hence, by the Sobolev imbedding theorem,

$$
\left(\int_{\Omega}\left(u_{n}^{+}\right)^{q} d x\right)^{p / q} \leq c \int_{\Omega}\left(\left|\nabla u_{n}^{+}\right|^{p}+\lambda g(x)\left(u_{n}^{+}\right)^{p}\right) d x \leq c \int_{\Omega}\left(u_{n}^{+}\right)^{q} d x
$$

and there exists $\nu>0$ such that $\int_{\Omega}\left(u_{n}^{+}\right)^{q} d x \geq \nu$. We have

$$
\int_{\Omega}\left(u^{+}\right)^{q} d x=\lim _{n \rightarrow \infty} \int_{\Omega}\left(u_{n}^{+}\right)^{q} d x \geq \nu .
$$

Hence $u^{+} \neq 0$. Similarly $u^{-} \neq 0$. Choose $s, t>0$ such that $s u^{+}-t u^{-} \in \Sigma_{q}$, that is $(s, t)$ solves the system

$$
\left\{\begin{array}{l}
s^{q} \cos \theta \int_{\Omega}\left(u^{+}\right)^{q} d x+t^{q} \sin \theta \int_{\Omega}\left(u^{-}\right)^{q} d x=1, \\
s^{p-q} R_{q}\left(u^{+}\right)=t^{p-q} R_{q}\left(-u^{-}\right) .
\end{array}\right.
$$

Let $a_{n}^{q}=G_{q}\left(s u_{n}^{+}-t u_{n}^{-}\right)$, then $G_{q}\left(s u_{n}^{+} / a_{n}-t u_{n}^{-} / a_{n}\right)=1$ and $a_{n} \rightarrow 1$ as $n \rightarrow \infty$. By the lemma below, we have

$$
\begin{aligned}
\mu_{q} \leq J\left(s u^{+}-t u^{-}\right) & \leq \lim _{n \rightarrow \infty} J\left(s u_{n}^{+}-t u_{n}^{-}\right) \\
& =\lim _{n \rightarrow \infty} J\left(\frac{s}{a_{n}} u_{n}^{+}-\frac{t}{a_{n}} u_{n}^{-}\right) \leq \lim _{n \rightarrow \infty} J\left(u_{n}^{+}-u_{n}^{-}\right)=\mu_{q} .
\end{aligned}
$$

Hence $J\left(s u^{+}-t u^{-}\right)=\mu_{q}$.

Lemma 3.3. Let $u \in \Sigma_{q}, u=u^{+}-u^{-}$. Let $s, t>0, v=s u^{+}-t u^{-}$and $G_{q}(v)=1$. Then $J(v) \leq J(u)$. 
Proof. Since $u \in \Sigma_{q}, G_{q}\left(u^{+}\right)+G_{q}\left(-u^{-}\right)=G_{q}(u)=1$,

$$
\frac{J\left(u^{+}\right)}{G_{q}\left(u^{+}\right)}=\frac{J\left(-u^{-}\right)}{G_{q}\left(-u^{-}\right)}=\frac{J(u)}{G_{q}(u)}=J(u) .
$$

We have

$$
\begin{aligned}
\frac{J(v)}{J(u)} & =\frac{s^{p} J\left(u^{+}\right)+t^{p} J\left(-u^{-}\right)}{J(u)}=s^{p} G_{q}\left(u^{+}\right)+t^{p} G_{q}\left(-u^{-}\right) \\
& \leq\left(s^{q} G_{q}\left(u^{+}\right)+t^{q} G_{q}\left(-u^{-}\right)\right)^{p / q}=\left(G_{q}\left(s u^{+}-t u^{-}\right)\right)^{p / q}=1 .
\end{aligned}
$$

LEMMA 3.4. The minimizer $u$ for $\mu_{q}$ solves the following equation:

$(\mathrm{E})_{q}$

$$
-\Delta_{p} u+\lambda g(x)|u|^{p-2} u=\mu_{q}\left(\cos \theta\left(u^{+}\right)^{q-1}-\sin \theta\left(u^{-}\right)^{q-1}\right)
$$

or in the weak form

$(\mathrm{E})_{q}$

$$
\begin{aligned}
\int_{\Omega}\left(|\nabla u|^{p-2} \nabla u \nabla \varphi+\lambda g(x)|u|^{p-2} u \varphi\right) d x & \\
& =\mu_{q} \int_{\Omega}\left(\cos \theta\left(u^{+}\right)^{q-1}-\sin \theta\left(u^{-}\right)^{q-1}\right) \varphi d x
\end{aligned}
$$

for all $\varphi \in W_{0}^{1, p}(\Omega)$.

Proof. We use an indirect argument. Suppose that the minimizer $u$ for problem $(\mathrm{M})_{q}$ does not satisfy $(\mathrm{E})_{q}$, then there exists a function $\varphi \in W_{0}^{1, p}(\Omega)$ such that

$\int_{\Omega}\left(|\nabla u|^{p-2} \nabla u \nabla \varphi+\lambda g(x)|u|^{p-2} u \varphi\right) d x<\mu_{q} \int_{\Omega}\left(\cos \theta\left(u^{+}\right)^{q-1}-\sin \theta\left(u^{-}\right)^{q-1}\right) \varphi d x$.

Replace $\varphi$ by $\varphi-u \int_{\Omega}\left(\cos \theta\left(u^{+}\right)^{q-1}-\sin \theta\left(u^{-}\right)^{q-1}\right) \varphi d x$, we may assume

$$
\begin{aligned}
\left(G_{q}^{\prime}(u), \varphi\right) & =\int_{\Omega}\left(\cos \theta\left(u^{+}\right)^{q-1}-\sin \theta\left(u^{-}\right)^{q-1}\right) \varphi d x=0, \\
\left(J^{\prime}(u), \varphi\right) & =\int_{\Omega}\left(|\nabla u|^{p-2} \nabla u \nabla \varphi+\lambda g(x)|u|^{p-2} u \varphi\right) d x<0 .
\end{aligned}
$$

Choose $\delta>0$ such that for $|s-1|<\delta,|t-1|<\delta$ and $0<\varepsilon<\delta$,

$$
\left(J^{\prime}\left(s u^{+}-t u^{-}+\varepsilon \varphi\right), \varphi\right) \leq-\frac{1}{2} .
$$

Consider the square $S$ on the $(s, t)$ plane with vertexes $A(1,1+\delta), B(1+\delta, 1)$, $C(1,1-\delta)$, and $D(1-\delta, 1)$, and edges $A B, B C, C D$ and $D A$. We have the following estimates:

$$
\begin{cases}G_{q}\left(s u^{+}-t u^{-}\right)>1 & \text { if }(s, t) \in A B, \\ G_{q}\left(s u^{+}-t u^{-}\right)<1 & \text { if }(s, t) \in C D, \\ R_{q}\left(s u^{+}\right)<R_{q}\left(-t u^{-}\right) & \text {if }(s, t) \in B C, \\ R_{q}\left(s u^{+}\right)>R_{q}\left(-t u^{-}\right) & \text {if }(s, t) \in D A .\end{cases}
$$


Choose $0<\varepsilon_{0}<\delta$ such that for $\varepsilon<\varepsilon_{0}$ we have, for $\omega_{\varepsilon}(s, t)=s u^{+}-t u^{-}+\varepsilon \varphi$,

$$
\begin{cases}G_{q}\left(\omega_{\varepsilon}(s, t)\right)>1 & \text { if }(s, t) \in A B, \\ G_{q}\left(\omega_{\varepsilon}(s, t)\right)<1 & \text { if }(s, t) \in C D, \\ R_{q}\left(\omega_{\varepsilon}^{+}(s, t)\right)<R_{q}\left(-\omega_{\varepsilon}^{-}(s, t)\right) & \text { if }(s, t) \in B C, \\ R_{q}\left(\omega_{\varepsilon}^{+}(s, t)\right)>R_{q}\left(-\omega_{\varepsilon}^{-}(s, t)\right) & \text { if }(s, t) \in D A .\end{cases}
$$

By a degree theorem argument [3], [9], for any $\varepsilon<\varepsilon_{0}$ we find $(s, t) \in S$ such that $\omega_{\varepsilon}(s, t)=s u^{+}-t u^{-}+\varepsilon \varphi \in \Sigma_{q}$. In fact the map

$$
T: S \ni(s, t) \mapsto\left(G_{q}\left(\omega_{\varepsilon}(s, t)\right)-1, R_{q}\left(\omega_{\varepsilon}^{+}(s, t)\right)-R_{q}\left(-\omega_{\varepsilon}^{-}(s, t)\right)\right)
$$

is homotopic to the map $K:(s, t) \mapsto(s+t-2, t-s)$. Hence

$$
\operatorname{deg}(T, \operatorname{int} S, 0)=\operatorname{deg}(K, \operatorname{int} S, 0)=1 .
$$

There exists $(\bar{s}, \bar{t}) \in S$ such that $T(\bar{s}, \bar{t})=(0,0)$, that is $\omega_{\varepsilon}(\bar{s}, \bar{t}) \in \Sigma_{q}$. Notice that $\bar{s} \rightarrow 1, \bar{t} \rightarrow 1$ as $\varepsilon \rightarrow 0$. We have

$G_{q}\left(\bar{s} u^{+}-\bar{t} u^{-}\right)=G_{q}\left(\bar{s} u^{+}-\bar{t} u^{-}+\varepsilon \varphi\right)-\varepsilon \int_{0}^{1}\left(G_{q}^{\prime}\left(\bar{s} u^{+}-\bar{t} u^{-}+\tau \varepsilon \varphi\right), \varphi\right) d \tau=1+o(\varepsilon)$.

Choose $\lambda>0$ such that $G_{q}\left(\lambda \bar{s} u^{+}-\lambda \bar{t} u^{-}\right)=1, \lambda=1+o(\varepsilon)$. Now, by Lemma 3.3,

$$
\begin{aligned}
J\left(\omega_{\varepsilon}(\bar{s}, \bar{t})\right) & =J\left(\bar{s} u^{+}-\bar{t} u^{-}+\varepsilon \varphi\right) \\
& =J\left(\bar{s} u^{+}-\bar{t} u^{-}\right)+\varepsilon \int_{0}^{1}\left(J^{\prime}\left(\bar{s} u^{+}-\bar{t} u^{-}+\tau \varepsilon \varphi\right), \varphi\right) d \tau \\
& \leq J\left(\bar{s} u^{+}-\bar{t} u^{-}\right)-\frac{1}{2} \varepsilon \leq J\left(\lambda \bar{s} u^{+}-\lambda \bar{t} u^{-}\right)-\frac{1}{4} \varepsilon \\
& \leq J\left(u^{+}-u^{-}\right)-\frac{1}{4} \varepsilon=\mu_{q}-\frac{1}{4} \varepsilon .
\end{aligned}
$$

We arrive at a contradiction.

LEMMA 3.5. $\varlimsup_{q \rightarrow p} \mu_{q} \leq \mu_{p}$.

Proof. Given $\varepsilon>0$, choose $u=u^{+}-u^{-} \in \Sigma_{p}$ with $J(u) \leq \mu_{p}+\varepsilon$. We are looking for $u_{q}=s_{q} u^{+}-t_{q} u^{-} \in \Sigma_{q}$ with $\lim _{q \rightarrow p} J\left(u_{q}\right)=J(u)$, then

$$
\varlimsup_{q \rightarrow p} \mu_{q} \leq \lim _{q \rightarrow p} J\left(u_{q}\right)=J(u) \leq \mu_{p}+\varepsilon,
$$

where $\varepsilon$ is arbitrary, hence $\varlimsup_{q \rightarrow p} \mu_{q} \leq \mu_{p}$. We solve the system for $\left(s_{q}, t_{q}\right)$,

$$
\left\{\begin{array}{l}
s_{q}^{q} G_{q}\left(u^{+}\right)+t_{q}^{q} G_{q}\left(u^{-}\right)=1, \\
\frac{s_{q}^{p} J\left(u^{+}\right)}{s_{q}^{q} G_{q}\left(u^{+}\right)}=\frac{t_{q}^{p} J\left(-u^{-}\right)}{t_{q}^{q} G_{q}\left(-u^{-}\right)} .
\end{array}\right.
$$


If $s_{q}>0, t_{q}>0$ is a solution, then $s_{q} u^{+}-t_{q} u^{-} \in \Sigma_{q}$. By the second equation of (3.1),

$$
a_{q}:=\frac{t_{q}}{s_{q}}=\left(\frac{G_{q}\left(u^{+}\right)}{J\left(u^{+}\right)} \frac{J\left(-u^{-}\right)}{G_{q}\left(-u^{-}\right)}\right)^{1 /(q-p)}=\left(\frac{\int_{\Omega}\left(u^{+}\right)^{q} d x}{\int_{\Omega}\left(u^{+}\right)^{p} d x} \frac{\int_{\Omega}\left(u^{-}\right)^{p} d x}{\int_{\Omega}\left(u^{-}\right)^{q} d x}\right)^{1 /(q-p)} .
$$

Define

$$
\begin{gathered}
a_{p}:=\lim _{q \rightarrow p} a_{q}=\exp \left\{\frac{\int_{\Omega}\left(u^{+}\right)^{p} \ln u^{+} d x}{\int_{\Omega}\left(u^{+}\right)^{p} d x}-\frac{\int_{\Omega}\left(u^{-}\right)^{p} \ln u^{-} d x}{\int_{\Omega}\left(u^{-}\right)^{p} d x}\right\} . \\
\left\{\begin{array}{l}
s_{q}=1 /\left\{\int_{\Omega}\left(\cos \theta\left(u^{+}\right)^{q}+\sin \theta\left(a_{q} u^{-}\right)^{q}\right) d x\right\}^{1 / q}, \\
t_{q}=a_{q} /\left\{\int_{\Omega}\left(\cos \theta\left(u^{+}\right)^{q}+\sin \theta\left(a_{q} u^{-}\right)^{q}\right) d x\right\}^{1 / q}
\end{array}\right. \\
\left\{\begin{array}{l}
s_{p}=1 /\left\{\int_{\Omega}\left(\cos \theta\left(u^{+}\right)^{p}+\sin \theta\left(a_{p} u^{-}\right)^{p}\right) d x\right\}^{1 / p}, \\
t_{p}=a_{p} /\left\{\int_{\Omega}\left(\cos \theta\left(u^{+}\right)^{p}+\sin \theta\left(a_{p} u^{-}\right)^{p}\right) d x\right\}^{1 / p} .
\end{array}\right.
\end{gathered}
$$

We have $\widetilde{u}=s_{p} u^{+}-t_{p} u^{-} \in \Sigma_{p}, J(\widetilde{u})=J(u) \leq \mu_{p}+\varepsilon$ and $u_{q}=s_{q} u^{+}-t_{q} u^{-} \in \Sigma_{q}$, $\lim _{q \rightarrow p} J\left(u_{q}\right)=J(\widetilde{u}) \leq \mu_{p}+\varepsilon$.

Proof of Theorem 3.1. Let $u_{q} \in \Sigma_{q}, q>p$, be the minimizer for problem $(\mathrm{M})_{q}$, obtained in Lemmas 3.3, 3.4. $J\left(u_{q}\right)=\mu_{q}, G_{q}\left(u_{q}\right)=1$ and $u_{q}$ satisfies

$$
-\Delta_{p} u_{q}+\lambda g(x)\left|u_{q}\right|^{p-2} u_{q}=\mu_{q}\left(\cos \theta\left(u^{+}\right)^{q-1}-\sin \theta\left(u^{-}\right)^{q-}\right)
$$

or in the weak form

$$
\begin{aligned}
\int_{\Omega}\left(\left|\nabla u_{q}\right|^{p-2} \nabla u_{q} \nabla \varphi+\lambda g(x)\left|u_{q}\right|^{p-2} u_{q} \varphi\right) d x \\
=\int_{\Omega} \mu_{q}\left(\cos \theta\left(u_{q}^{+}\right)^{q-1}-\sin \theta\left(u_{q}^{-}\right)^{q-1}\right) \varphi d x
\end{aligned}
$$

for all $\varphi \in W_{0}^{1, p}(\Omega)$. Choose a sequence $\left\{q_{n}\right\}, q_{n} \rightarrow p$, and

$$
\mu=\lim _{n \rightarrow \infty} \mu_{q_{n}}=\lim _{q \rightarrow p} \mu_{q} .
$$

Denote $u_{n}=u_{q_{n}}, J\left(u_{n}\right)=\mu_{q_{n}} \leq c,\left\{u_{n}\right\}$ is bounded in $W_{0}^{1, p}(\Omega)$. Assume $u_{n} \rightarrow u$ in $L^{r}(\Omega), p \leq r<p^{*}$. Denote $f_{n}=\mu_{q}\left(\cos \theta\left(u_{n}^{+}\right)^{q_{n}-1}-\sin \theta\left(u_{n}^{-}\right)^{q_{n}-1}\right)$, suppose $f_{n}$ is bounded in $L^{r /(r-1)}(\Omega)$, for $p \leq r<p^{*}$. 
For $p \geq 2$, we have

$$
\begin{gathered}
\int_{\Omega}\left|\nabla u_{n}-\nabla u_{m}\right|^{p} d x \leq c \int_{\Omega}\left(\left|\nabla u_{n}\right|^{p-2} \nabla u_{n}-\left|\nabla u_{m}\right|^{p-2} \nabla u_{m}\right)\left(\nabla u_{n}-\nabla u_{m}\right) d x \\
\quad \leq c \int_{\Omega}\left(f_{n}-f_{m}\right)\left(u_{n}-u_{m}\right) d x \leq c\left(\left|f_{n}\right|_{r /(r-1)}+\left|f_{m}\right|_{r /(r-1)}\right)\left|u_{n}-u_{m}\right|_{r} \rightarrow 0 .
\end{gathered}
$$

For $1<p \leq 2$, we have

$$
\begin{aligned}
& \int_{\Omega}\left|\nabla u_{n}-\nabla u_{m}\right|^{p} d x \\
& \leq c\left(\int_{\Omega}\left(\left|\nabla u_{n}\right|^{p-2} \nabla u_{n}-\left|\nabla u_{m}\right|^{p-2} \nabla u_{m}\right)\left(\nabla u_{n}-\nabla u_{m}\right) d x\right)^{p / 2} \\
& \cdot\left(\int_{\Omega}\left(\left|\nabla u_{n}\right|^{p}+\left|\nabla u_{m}\right|^{p}\right) d x\right)^{(2-p) / 2} \\
& \leq c\left|\int_{\Omega}\left(f_{n}-f_{m}\right)\left(u_{n}-u_{m}\right) d x\right|^{p / 2} \leq c\left|u_{n}-u_{m}\right|_{r}^{p / 2} \rightarrow 0 .
\end{aligned}
$$

In both cases we have $u_{n} \rightarrow u$ in $W_{0}^{1, p}(\Omega)$. Hence $G(u)=1, u \neq 0$, and $u$ satisfies the equation

$$
-\Delta_{p} u+\lambda g(x)|u|^{p-2} u=\mu\left(\cos \theta\left(u^{+}\right)^{p-1}-\sin \theta\left(u^{-}\right)^{p-1}\right)
$$

or in the weak form

$$
\begin{aligned}
\int_{\Omega}\left(|\nabla u|^{p-2} \nabla u \nabla \varphi+\lambda g(x)|u|^{p-2} u \varphi\right) d x & \\
& =\mu \int_{\Omega}\left(\cos \theta\left(u^{+}\right)^{p-1}-\sin \theta\left(u^{-}\right)^{p-1}\right) \varphi d x,
\end{aligned}
$$

for all $\varphi \in W_{0}^{1, p}(\Omega)$. We claim $u^{ \pm} \neq 0$, hence $u \in \Sigma_{p}$. Otherwise suppose $u^{-}=0$. Then $u$ is a nonnegative eigenfunction of the operator $\left(L_{\lambda}, W_{0}^{1, p}(\Omega)\right)$. It is strictly positive in $\Omega$ [7]. Hence $\left|u_{n}^{-}>0\right| \rightarrow 0$, where $\left|u_{n}^{-}>0\right|$ denotes the measure of the set $\left\{x \mid u_{n}^{-}>0\right\}$. On the other hand,

$$
\begin{aligned}
\left\|u_{n}^{-}\right\|_{\lambda}^{p} & =\int_{\Omega}\left(\left|\nabla u_{n}^{-}\right|^{p}+\lambda g(x)\left(u_{n}^{-}\right)^{p}\right) d x \\
= & \mu_{q_{n}} \int_{\Omega} \sin \theta\left(u_{n}^{-}\right)^{q_{n}} d x \\
& \leq c\left|u_{n}^{-}>0\right|^{1-q_{n} / p^{*}}\left(\int_{\Omega}\left(u_{n}^{-}\right)^{p^{*}} d x\right)^{q_{n} / p^{*}} \\
& \leq c\left|u_{n}^{-}>0\right|^{1-r / p^{*}}\left\|u_{n}^{-}\right\|_{\lambda}^{p}
\end{aligned}
$$

we arrive at a contradiction. Since $u \in \Sigma_{p}$, by (3.2) and Lemma 3.5,

$$
\varlimsup_{q \rightarrow p} \mu_{q} \leq \mu_{p} \leq J(u)=\mu=\lim _{q \rightarrow p} \mu_{q} .
$$

Hence $\mu=\mu_{p}$. 
LEMMA 3.6. The following holds:

(a) $\rho(\theta)=\bar{\rho}(\theta):=\inf \left\{\max \left(R\left(u^{+}\right), R\left(-u^{-}\right)\right) \mid u=u^{+}-u^{-} \in W^{1, p}\left(\mathbb{R}^{N}\right)\right.$ $\left.u^{ \pm} \neq 0\right\}$,

$\mu_{p}=\bar{\mu}_{p}:=\inf \left\{\max \left(R\left(u^{+}\right), R\left(-u^{-}\right)\right) \mid u=u^{+}-u^{-} \in W_{0}^{1, p}(\Omega), u^{ \pm} \neq 0\right\}$.

(b) $\lim _{R \rightarrow \infty} \mu_{p}\left(B_{R}\right)=\rho(\theta)$.

(c) If $\lambda>\Gamma(\theta)(0<\theta \leq \pi / 4)$, then $\rho(\theta) \cos \theta<\lambda$.

Proof. (a) $R$ is homogeneous, hence

$$
\rho(\theta)=\inf \left\{R(u) \mid u=u^{+}-u^{-} \in W^{1, p}\left(\mathbb{R}^{N}\right), u^{ \pm} \neq 0, R\left(u^{+}\right)=R\left(-u^{-}\right)\right\}
$$

and $\bar{\rho}(\theta) \leq \rho(\theta)$. On the other hand, let $u=u^{+}-u^{-} \in W^{1, p}\left(\mathbb{R}^{N}\right), u^{ \pm} \neq 0$. Suppose $R\left(u^{+}\right) \leq R\left(-u^{-}\right)$. Let $x_{0}$ be a Lebesgue point of $u, u\left(x_{0}\right)>0$, let $\varphi_{r}$ be a cut off function such that $\varphi_{r}=1,\left|x-x_{0}\right| \leq r, \varphi=0,\left|x-x_{0}\right| \geq 2 r$. Let $v_{r}=u^{+} \varphi_{r}-u^{-}$. Since $R\left(u^{+} \varphi_{r}\right) \rightarrow R\left(u^{+}\right)$as $r \rightarrow+\infty$, and $R\left(u^{+} \varphi_{r}\right) \rightarrow+\infty$ as $r \rightarrow 0$, there exists $r_{0}$ such that $R\left(u^{+} \varphi_{r_{0}}\right)=R\left(u^{-}\right)$, we have

$$
\rho(\theta) \leq R\left(v_{r_{0}}\right)=R\left(u^{-}\right)=\max \left(R\left(u^{+}\right), R\left(u^{-}\right)\right)
$$

hence $\rho(\theta) \leq \bar{\rho}(\theta)$. Similarly we have $\mu_{p}=\bar{\mu}_{p}$.

(b) It is clear that $\mu_{p}\left(B_{R}\right) \geq \rho(\theta)$ and $\mu_{p}\left(B_{R}\right)$ is nonincreasing in $R$, hence $\lim _{R \rightarrow \infty} \mu_{p}\left(B_{R}\right) \geq \rho(\theta)$. Given $\varepsilon>0$, choose $u \in \Sigma$ with $J(u) \leq \rho(\theta)+\varepsilon$. Let $\psi_{R}$ be a cut off function such that $\psi_{R}=1$, if $|x| \leq R ; \psi_{R}=0$, if $|x| \geq R+1$. For $R_{0}$ large enough,

$$
R\left(u^{+} \psi_{R}\right) \leq R\left(u^{+}\right)+\varepsilon \leq R(u)+\varepsilon=J(u)+\varepsilon .
$$

Similarly, $R_{p}\left(-u^{-} \psi_{R_{0}}\right) \leq J(u)+\varepsilon$ and

$$
\mu_{p}\left(B_{R}\right) \leq \max \left(R\left(u^{+} \psi_{R}\right), R\left(-u^{-} \psi_{R}\right)\right) \leq R(u)+\varepsilon=J(u)+\varepsilon \leq \rho(\theta)+2 \varepsilon .
$$

(c) There exists $\varepsilon>0$, choose $u \in W^{1, p}\left(\mathbb{R}^{N}\right)$, such that $u=u^{+}-u^{-}, u^{ \pm} \neq 0$ and

$$
\frac{\int_{\mathbb{R}^{N}}\left|\nabla u^{+}\right|^{p} d x}{\int_{\mathbb{R}^{N}}(1-g(x))\left(u^{+}\right)^{p} d x}=\frac{\int_{\mathbb{R}^{N}}\left|\nabla u^{-}\right|^{p} d x}{\int_{\mathbb{R}^{N}}(\tan \theta-g(x))\left(u^{-}\right)^{p} d x}<\lambda,
$$

hence

$$
\begin{array}{r}
R\left(u^{+}\right)=\frac{\int_{\mathbb{R}^{N}}\left(\left|\nabla u^{+}\right|^{p}+\lambda g(x)\left(u^{+}\right)^{p}\right) d x}{\cos \theta \int_{\mathbb{R}^{N}}\left(u^{+}\right)^{p} d x}<\frac{\lambda}{\cos \theta}, \\
R\left(-u^{-}\right)=\frac{\int_{\mathbb{R}^{N}}\left(\left|\nabla u^{-}\right|^{p}+\lambda g(x)\left(u^{-}\right)^{p}\right) d x}{\sin \theta \int_{\mathbb{R}^{N}}\left(u^{-}\right)^{p} d x}<\frac{\lambda}{\cos \theta},
\end{array}
$$




$$
\rho(\theta) \leq \max \left(R\left(u^{+}\right), R\left(-u^{-}\right)\right)<\frac{\lambda}{\cos \theta} .
$$

Proof of Theorem 1.2. Let $R_{n} \rightarrow \infty, \mu_{n}=\mu_{p}\left(B_{R_{n}}\right), u_{n} \in \Sigma_{p}\left(B_{R_{n}}\right)=$ $\Sigma \cap W_{0}^{1, p}(\Omega)$ satisfy

$$
-\Delta_{p} u_{n}+\lambda g(x)\left|u_{n}\right|^{p-2} u_{n}=\mu_{n}\left(\cos \theta\left(u_{n}^{+}\right)^{p-1}-\sin \theta\left(u_{n}^{-}\right)^{p-1}\right)
$$

or

$$
\begin{aligned}
\int_{B_{R_{n}}}\left(\left|\nabla u_{n}\right|^{p-2} \nabla u_{n} \nabla \varphi+\lambda g(x)\left|u_{n}\right|^{p-2} u_{n} \varphi\right) d x & \\
= & \mu_{n} \int_{B_{R_{n}}}\left(\cos \theta\left(u_{n}^{+}\right)^{p-1}-\sin \theta\left(u_{n}^{-}\right)^{p-1}\right) \varphi d x
\end{aligned}
$$

for all $\varphi \in W_{0}^{1, p}\left(B_{R_{n}}\right)$. By Lemma 3.6,

$$
\int_{B_{R_{n}}}\left(\left|\nabla u_{n}\right|^{p}+\lambda g(x)\left|u_{n}\right|^{p}\right) d x=\mu_{n} \rightarrow \rho(\theta) .
$$

We assume that $u_{n} \rightarrow u$ in $W^{1, p}\left(\mathbb{R}^{N}\right), u_{n} \rightarrow u$ in $L_{\mathrm{loc}}^{r}\left(\mathbb{R}^{N}\right), p \leq r<p^{*}$. By the assumption $\lambda>\Gamma(\theta)$ and Lemma 3.6, there exist $R, \delta, \nu, N$ such that

$$
\lambda g(x)-\mu_{n} \cos \theta \geq \delta>0, \quad \text { for }|x| \geq R \text { and } n \geq N .
$$

We have

$$
\begin{aligned}
\mu_{n} \int_{B_{R}} & \left(\cos \theta\left(u_{n}^{+}\right)^{p}+\sin \theta\left(u_{n}^{-}\right)^{p}\right) d x=\int_{\mathbb{R}^{N}}\left|\nabla u_{n}\right|^{p} d x+\int_{B_{R}} \lambda g(x)\left|u_{n}\right|^{p} d x \\
& +\int_{\mathbb{R}^{N} \backslash B_{R}}\left[\left(\lambda g(x)-\mu_{n} \cos \theta\right)\left(u_{n}^{+}\right)^{p}+\left(\lambda g(x)-\mu_{n} \sin \theta\right)\left(u_{n}^{-}\right)^{p}\right] d x \\
\geq & \int_{\mathbb{R}^{N}}\left|\nabla u_{n}\right|^{p} d x+\int_{B_{R}} \lambda g(x)\left|u_{n}\right|^{p} d x+\delta \int_{\mathbb{R}^{N} \backslash B_{R}}\left|u_{n}\right|^{p} d x \geq \nu J\left(u_{n}\right)=\nu \mu_{n}
\end{aligned}
$$

and

$\int_{B_{R}}\left(\cos \theta\left(u^{+}\right)^{p}+\sin \theta\left(u^{-}\right)^{p}\right) d x=\lim _{n \rightarrow \infty} \int_{B_{R}}\left(\cos \theta\left(u_{n}^{+}\right)^{p}+\sin \theta\left(u_{n}^{-}\right)^{p}\right) d x \geq \nu>0$.

Hence $u \neq 0$. As in the proof of Theorem 3.1, we can show that $u_{n} \rightarrow u$ in $W_{\text {loc }}^{1, p}\left(\mathbb{R}^{N}\right)$ and $u$ satisfies the equation

$$
-\Delta_{p} u+\lambda g(x)|u|^{p-2} u=\rho(\theta)\left(\cos \theta\left(u^{+}\right)^{p-1}-\sin \theta\left(u^{-}\right)^{p-1}\right)
$$

or, for all $\varphi \in W^{1, p}\left(\mathbb{R}^{N}\right)$

$$
\begin{aligned}
\int_{\mathbb{R}^{N}}\left(|\nabla u|^{p-2} \nabla u \nabla \varphi+\lambda g(x)|u|^{p-2} u \varphi\right) d x & \\
& =\rho(\theta) \int_{\mathbb{R}^{N}}\left(\cos \theta\left(u^{+}\right)^{p-1}-\sin \theta\left(u^{-}\right)^{p-1}\right) \varphi d x .
\end{aligned}
$$

Now we prove that $u^{ \pm} \neq 0$. Otherwise suppose $u^{-}=0$. Then $u=u^{+}$is a nonnegative eigenfunction of the operator $L_{\lambda}$, it should be strictly positive, 
see [8]. Therefore $\left|B_{R}\left(u_{n}^{-}\right)>0\right| \rightarrow 0$, where $B_{R}\left(u_{n}^{-}>0\right)=B_{R} \cap\left\{u_{n}^{-}>0\right\}$. As in (3.3), we have, on one hand,

$$
\begin{aligned}
\mu_{n} \sin & \theta \int_{B_{R}}\left(u_{n}^{-}\right)^{p} d x \\
& =\int_{\mathbb{R}^{N}}\left|\nabla u_{n}^{-}\right|^{p} d x+\int_{B_{R}} \lambda g(x)\left|u_{n}^{-}\right|^{p} d x+\int_{\mathbb{R}^{N} \backslash B_{R}}\left(\lambda g(x)-\mu_{n} \sin \theta\right)\left(u_{n}^{-}\right)^{p} d x \\
& \geq \int_{\mathbb{R}^{N}}\left|\nabla u_{n}^{-}\right|^{p} d x+\int_{B_{R}} \lambda g(x)\left|u_{n}^{-}\right|^{p} d x+\delta \int_{\mathbb{R}^{N} \backslash B_{R}}\left(u_{n}^{-}\right)^{p} d x \geq c\left\|u_{n}^{-}\right\|^{p} .
\end{aligned}
$$

On the other hand,

$$
\begin{aligned}
\mu_{n} \sin \theta \int_{B_{R}}\left(u_{n}^{-}\right)^{p} d x \leq c\left|B_{R}\left(u_{n}^{-}>0\right)\right|^{p / N}\left(\int_{B_{R}}\right. & \left.\left(u_{n}^{-}\right)^{p^{*}}\right)^{p / p^{*}} \\
& \leq c\left|B_{R}\left(u_{n}^{-}>0\right)\right|^{p / N}\left\|u_{n}^{-}\right\|^{p},
\end{aligned}
$$

we arrive at a contradiction. Similarly $u^{+} \neq 0$. It is clear that $J(u)=\rho(\theta)$ and $R\left(u^{+}\right)=R\left(-u^{-}\right)$.

Proposition 3.7. $\rho(\theta)$ is continuous.

Proof. Assume $0<\theta_{1}<\theta_{2}<\pi / 2$. Choose $u \in W^{1, p}\left(\mathbb{R}^{N}\right)$ such that

$$
\frac{\int_{\mathbb{R}^{N}}\left(\left|\nabla u^{+}\right|^{p}+\lambda g(x)\left(u^{+}\right)^{p}\right) d x}{\cos \theta_{1} \int_{\mathbb{R}^{N}}\left(u^{+}\right)^{p} d x}=\frac{\int_{\mathbb{R}^{N}}\left(\left|\nabla u^{-}\right|^{p}+\lambda g(x)\left(u^{-}\right)^{p}\right) d x}{\sin \theta_{1} \int_{\mathbb{R}^{N}}\left(u^{-}\right)^{p} d x} \leq \rho\left(\theta_{1}\right)+\varepsilon,
$$

we have

$$
\begin{aligned}
& \frac{\int_{\mathbb{R}^{N}}\left(\left|\nabla u^{+}\right|^{p}+\lambda g(x)\left(u^{+}\right)^{p}\right) d x}{\cos \theta_{2} \int_{\mathbb{R}^{N}}\left(u^{+}\right)^{p} d x} \leq\left(\rho\left(\theta_{1}\right)+\varepsilon\right) \frac{\cos \theta_{1}}{\cos \theta_{2}}, \\
& \frac{\int_{\mathbb{R}^{N}}\left(\left|\nabla u^{-}\right|^{p}+\lambda g(x)\left(u^{-}\right)^{p}\right) d x}{\sin \theta_{2} \int_{\mathbb{R}^{N}}\left(u^{-}\right)^{p} d x} \leq\left(\rho\left(\theta_{1}\right)+\varepsilon\right) \frac{\sin \theta_{1}}{\sin \theta_{2}}<\left(\rho\left(\theta_{1}\right)+\varepsilon\right) \frac{\cos \theta_{1}}{\cos \theta_{2}} .
\end{aligned}
$$

By Lemma 3.6 (a)

$$
\begin{aligned}
\rho\left(\theta_{2}\right) \leq \max \left\{\frac{\int_{\mathbb{R}^{N}}\left(\left|\nabla u^{+}\right|^{p}+\lambda g(x)\left(u^{+}\right)^{p}\right) d x}{\cos \theta_{2} \int_{\mathbb{R}^{N}}\left(u^{+}\right)^{p} d x},\right. \\
\left.\frac{\int_{\mathbb{R}^{N}}\left(\left|\nabla u^{-}\right|^{p}+\lambda g(x)\left(u^{-}\right)^{p}\right) d x}{\sin \theta_{2} \int_{\mathbb{R}^{N}}\left(u^{-}\right)^{p} d x}\right\} \leq\left(\rho\left(\theta_{1}\right)+\varepsilon\right) \frac{\cos \theta_{1}}{\cos \theta_{2}},
\end{aligned}
$$


where $\varepsilon$ is arbitrary,

$$
\rho\left(\theta_{2}\right) \cos \theta_{2} \leq \rho\left(\theta_{1}\right) \cos \theta_{1}, \quad 0<\theta_{1}<\theta_{2}<\frac{\pi}{2} .
$$

Hence

$$
\varlimsup_{\theta \rightarrow \theta_{0}^{+}} \rho(\theta) \leq \rho\left(\theta_{0}^{+}\right) \leq \lim _{\theta \rightarrow \theta_{0}^{+}} \rho(\theta) .
$$

Now choose $u \in W^{1, p}\left(\mathbb{R}^{N}\right)$ such that

$$
\frac{\int_{\mathbb{R}^{N}}\left(\left|\nabla u^{+}\right|^{p}+\lambda g(x)\left(u^{+}\right)^{p}\right) d x}{\cos \theta_{2} \int_{\mathbb{R}^{N}}\left(u^{+}\right)^{p} d x}=\frac{\int_{\mathbb{R}^{N}}\left(\left|\nabla u^{-}\right|^{p}+\lambda g(x)\left(u^{-}\right)^{p}\right) d x}{\sin \theta_{2} \int_{\mathbb{R}^{N}}\left(u^{-}\right)^{p} d x} \leq \rho\left(\theta_{2}\right)+\varepsilon .
$$

We have

$$
\begin{aligned}
& \frac{\int_{\mathbb{R}^{N}}\left(\left|\nabla u^{-}\right|^{p}+\lambda g(x)\left(u^{-}\right)^{p}\right) d x}{\sin \theta_{1} \int_{\mathbb{R}^{N}}\left(u^{-}\right)^{p} d x} \leq\left(\rho\left(\theta_{2}\right)+\varepsilon\right) \frac{\sin \theta_{2}}{\sin \theta_{1}}, \\
& \frac{\int_{\mathbb{R}^{N}}\left(\left|\nabla u^{+}\right|^{p}+\lambda g(x)\left(u^{+}\right)^{p}\right) d x}{\cos \theta_{1} \int_{\mathbb{R}^{N}}\left(u^{+}\right)^{p} d x} \leq\left(\rho\left(\theta_{2}\right)+\varepsilon\right) \frac{\cos \theta_{2}}{\cos \theta_{1}}<\left(\rho\left(\theta_{2}\right)+\varepsilon\right) \frac{\sin \theta_{2}}{\sin \theta_{1}} .
\end{aligned}
$$

Again by Lemma $3.6(1), \rho\left(\theta_{1}\right) \sin \theta_{1} \leq \rho\left(\theta_{2}\right) \sin \theta_{2}$. Hence

$$
\varlimsup_{\theta \rightarrow \theta_{0}^{-}} \rho(\theta) \leq \rho\left(\theta_{0}\right) \leq \lim _{\theta \rightarrow \theta_{0}^{-}} \rho(\theta) .
$$

By (3.4) and (3.5), $\lim _{\theta \rightarrow \theta_{0}} \rho(\theta)=\rho\left(\theta_{0}\right)$.

\section{REFERENCES}

[1] T. Bartsch, Z. LiU AND T. WETh, Sign-changing solutions of superlinear Schrödinger equations, Comm. Partial Differential Equations 29 (1-2) (2004), 25-42.

[2] T. Bartsch, Z.-Q. WANG AND Z.T. ZhANG, On the Fučik point spectrum for Schrödinger operators on $\mathbb{R}^{N}$, J. Fixed Point Theory Appl. 5 (2009), 305-317.

[3] G. Cerami, S. Solimini and M. Struwe, Some existence results for superlinear elliptic boundary value problems involving critical exponents, J. Funct. Anal. 69 (1986), 289-306.

[4] M. Cuesta, D. De Figueiredo And J.-P.Gossez, The beginning of the Fučik spectrum for p-Laplacian, J. Differential Equations 159 (1999), 212-238.

[5] S. FUČIK, Solvability of nonlinear equations and boundary value problems, D. Reidel Publishing Company, Dordrecht, 1980.

[6] Y.X. Guo AND X.Q. LiU, A multiple critical points theorem and applications to quasilinear boundary value problems in $\mathbb{R}_{+}^{N}$, Nonlinear Anal. 75 (2012), 3787-3808.

[7] P. Lindqvist, On the equation $\operatorname{div}\left(|\nabla u|^{p-2} \nabla u\right)+\lambda|u|^{p-2} u=0$, Proc. Amer. Math. Soc. 109 (1) (1990), 157-164.

[8] J.Q. LIU, X.Q. LiU AND Y.X. GuO, On an asymptotically p-linear p-Laplacian equation in $\mathbb{R}^{N}$, Nonliear Anal. 74 (2011), 676-688. 
[9] J.Q. LiU, Y.Q. WANG AND Z.-Q. WANG, Solutions for quasilinear Schrödinger equations via the Nehari method, Comm. Partial Differential Equations 29 (5-6) (2004), 879-901.

[10] X.Q. LIU AND Y.X. GuO, Sign-changing solutions for an asymptotically p-linear pLaplacian equations in $\mathbb{R}^{N}$, Comm. Contemp. Math. 15 (1) (2013), 1250046 (24 pages).

[11] X.Q. LiU, Y.S. HuANG AND J.Q. LiU, Sign-changing solutions for an asymptotically linear Schrödinger equation with deepening potential well, Adv. Differential Equations 16 (1-2) (2011), 1-30.

[12] Z. LiU AND J.X. Sun, Invariant sets of descending flow in the critical point theory with applications to nonlinear differential equations, J. Differential Equations 172 (2001), 257299.

[13] Z. LiU AND Z.-Q. WANG, Sign-changing solutions of nonlinear elliptic equations, Front. Math. China 3 (2008), 221-238.

[14] M. Struwe, A global compactness result for elliptic boundary value problems involving limiting nonlinearities, Math. Z. 187 (1984), 511-517.

[15] C. Stuart And H.S. Zou, Global branch of solutions for nonlinear Schrödinger equations with deepening potential well, Proc. London Math. Soc. 92 (2006), 655-681.

[16] Z.P. WANG AND H.S. Zou, Positive solutions for nonlinear Schrödinger equations with deepening potential well, J. Eur. Math. Soc. 11 (2009), 545-573.

[17] X.P. ZhU AND D.M. CAO, The concentration-compactness principle in nonlinear elliptic equations, Acta. Math. Sci. 9 (1989), 307-328.

Ming Xiong and Ze-Heng Yang

Department of Mathematics

Dali University

Dali 671000, P.R. CHINA

XiAng-QIng LiU

Department of Mathematics

Yunnan Normal University

Kunming 650000, P.R. CHINA

E-mail address: lxq8u8@163.com 